\title{
CORETRACTABLE MODULES
}

\author{
B. AMINI ${ }^{凶}$, M. ERSHAD and H. SHARIF
}

(Received 18 February 2007; accepted 1 November 2007)

Communicated by J. Du

\begin{abstract}
An $R$-module $M$ is called coretractable if $\operatorname{Hom}_{R}(M / K, M) \neq 0$ for any proper submodule $K$ of $M$. In this paper we study coretractable modules and their endomorphism rings. It turns out that if all right $R$-modules are coretractable, then $R$ is a right Kasch and (two-sided) perfect ring. However, the converse holds for commutative rings. Also, for a semi-injective coretractable module $M_{R}$ with $S=\operatorname{End}_{R}(M)$, we show that $\mathrm{u} \cdot \operatorname{dim}\left({ }_{S} S\right)=\operatorname{corank}\left(M_{R}\right)$.
\end{abstract}

2000 Mathematics subject classification: primary 16D10; secondary 16D40, 16L30.

Keywords and phrases: coretractable module, Kasch ring, perfect ring, semi-Artinian ring, max ring, semi-injective module.

\section{Introduction}

Throughout this paper, all rings are associative with identity and all modules are unitary right modules unless stated otherwise. Homomorphisms between modules are written and composed on the opposite side of scalars. Semisimple rings are in the sense of Wedderburn and Artin: these are rings which are semisimple as right (or left) modules over themselves. By a regular ring, we mean a ring $R$ such that $x \in x R x$ for any $x \in R$ (that is, a von Neumann regular ring). For a module $M_{R}$ we write $\operatorname{Soc}\left(M_{R}\right), \operatorname{Rad}\left(M_{R}\right)$ and $\mathrm{E}\left(M_{R}\right)$ for the socle, the Jacobson radical and the injective hull of $M_{R}$, respectively. Also $\mathrm{J}(R)$ will be used for the Jacobson radical of a ring $R$. The notation $K \leq M, K \ll M$ and $K \unlhd M$ will denote that $K$ is a submodule, a superfluous submodule and an essential submodule of $M$, respectively.

Let $S$ be a ring and let ${ }_{S} M$ be a left $S$-module. Then for any $X \subseteq M$ and $Y \subseteq S$, the left annihilator of $X$ in $S$ and the right annihilator of $Y$ in $M$ are

$$
l_{S}(X)=\{s \in S \mid s x=0 \text { for all } x \in X\}
$$

(C) 2009 Australian Mathematical Society 1446-7887/2009 \$16.00 
and

$$
r_{M}(Y)=\{m \in M \mid y m=0 \text { for all } y \in Y\},
$$

respectively. Also for a right $R$-module $N_{R}, r_{R}(Z)$ and $l_{N}(W)$ are defined similarly for any $Z \subseteq N$ and $W \subseteq R$.

Following Khuri [5], an $R$-module $M$ is said to be retractable if $\operatorname{Hom}_{R}(M, N) \neq 0$ for any nonzero submodule $N$ of $M$. Retractable modules have been investigated by some authors (see, for example, [4-6, 9, 13, 14]). Dually an $R$-module $M$ is called coretractable if $\operatorname{Hom}_{R}(M / K, M) \neq 0$ for any proper submodule $K$ of $M$. In Section 2 we study some properties of coretractable modules. We show that coretractability is preserved under finite direct sums. Also we observe that a ring $R$ is right Kasch if and only if $R_{R}$ is coretractable, but for modules, coretractability and being Kasch are not equivalent conditions. In Section 3, we call a ring $R$ right CC (completely coretractable) if all right $R$-modules are coretractable. It is proved that right $\mathrm{CC}$ rings are two-sided perfect. However, an Artinian ring need not be right CC. Also we find conditions under which free modules are coretractable. Section 4 is concerned with the endomorphism rings of coretractable modules. For a coretractable module $M_{R}$ with $S=\operatorname{End}_{R}(M)$ we show that $S$ is a regular ring if and only if $M_{R}$ is semisimple. Moreover, if $M_{R}$ is semi-injective, then $\mathrm{u} \cdot \operatorname{dim}\left({ }_{S} S\right)=\operatorname{corank}\left(M_{R}\right)$.

\section{Coretractable modules}

It is natural to consider the dual notion of retractable modules. In this section we define the concept of coretractability for modules.

Definition 2.1. An $R$-module $M$ is called coretractable if, for any proper submodule $K$ of $M$, there exists a nonzero homomorphism $f: M \longrightarrow M$ with $f(K)=0$, that is, $\operatorname{Hom}_{R}(M / K, M) \neq 0$.

One can easily see that an $R$-module $M$ is coretractable if and only if $\operatorname{Hom}_{R}(M / K, M) \neq 0$ for any proper essential submodule $K$ of $M$. (For if $L$ is a submodule of $M$, then there exists $L^{\prime} \leq M$ such that $L \oplus L^{\prime} \unlhd M$.)

EXAMPLE 2.2. (a) Clearly semisimple modules and cogenerators (in the category Mod- $R$ ) are coretractable.

(b) The Prüfer $p$-group $\mathbb{Z}_{p^{\infty}}$ is a coretractable $\mathbb{Z}$-module (since, for any proper submodule $K$ of $\mathbb{Z}_{p^{\infty}}, \mathbb{Z}_{p^{\infty}} / K \cong \mathbb{Z}_{p^{\infty}}$ ).

(c) Since $\operatorname{Hom}_{\mathbb{Z}}(\mathbb{Q} / \mathbb{Z}, \mathbb{Q})=0$, the $\mathbb{Z}$-module $\mathbb{Q}$ is not coretractable.

(d) Let $n>1$ be a positive integer. It is easy to check that the $\mathbb{Z}$-module $\mathbb{Z}_{n}=\mathbb{Z} / n \mathbb{Z}$ is coretractable.

We now obtain some properties of coretractable modules.

Proposition 2.3. Let $M$ be a coretractable R-module.

(a) If $\operatorname{Rad}(M) \neq M$, then $\operatorname{Soc}(M) \neq 0$.

(b) If $M$ is nonsingular, then it is semisimple. 
Proof. (a) Let $K$ be a maximal submodule of $M$. Since $\operatorname{Hom}_{R}(M / K, M) \neq 0$ and $M / K$ is a simple $R$-module, $\operatorname{Soc}(M) \neq 0$.

(b) Suppose on the contrary that $M$ is not semisimple. So $M$ has a proper essential submodule $K$. Since $M / K$ is singular and $M$ is nonsingular, $\operatorname{Hom}_{R}(M / K, M)=0$, which is a contradiction.

Since over a right nonsingular ring any projective right module is nonsingular, the following result is an immediate consequence of Proposition 2.3.

COROLLARY 2.4. Let $R$ be a right nonsingular ring. Then a projective $R$-module is coretractable if and only if it is semisimple.

Let $R$ be a ring and let $C$ be a cogenerator. Then for any module $M, C \oplus M$ is a cogenerator and so is a coretractable module. But $M$ need not be coretractable. Therefore, being coretractable is not preserved by taking submodules, factor modules and direct summands. However, there are some special cases, as follows.

Proposition 2.5. Let $M=K \oplus L$ be a coretractable module. If $K$ is a fully invariant submodule of $M$ or $K$ cogenerates $M$, then $K$ is also coretractable. In particular, if $\bigoplus_{I} K$ or $\prod_{I} K$ is coretractable for some index set $I$, then so is $K$.

PROOF. Let $U$ be a proper submodule of $K$. There exists $0 \neq f \in \operatorname{End}_{R}(M)$ with $f(U \oplus L)=0$. If $K$ is a fully invariant submodule of $M$, then

$$
0 \neq\left. f\right|_{K} \in \operatorname{End}_{R}(K) \text { and }\left(\left.f\right|_{K}\right)(U)=0 .
$$

Now suppose that $K$ cogenerates $M$. So there exists $g \in \operatorname{Hom}_{R}(M, K)$ with $g f \neq 0$. Thus $0 \neq\left. g f\right|_{K} \in \operatorname{End}_{R}(K)$ and $\left(\left.g f\right|_{K}\right)(U)=0$. So in both cases $K$ is coretractable.

PROpOSition 2.6. If $M_{1}, M_{2}, \ldots, M_{n}$ are coretractable $R$-modules, then so is $M=\bigoplus_{i=1}^{n} M_{i}$.

ProOf. Let $K$ be a proper submodule of $M$. There exists a least integer $j$ with $K+\left(M_{1} \oplus \cdots \oplus M_{j}\right)=M$. Let

$$
L=K+\left(M_{1} \oplus \cdots \oplus M_{j-1}\right) \quad \text { if } j>1 \quad \text { and } \quad L=K \quad \text { if } j=1 .
$$

So $L \neq M$ and $L \cap M_{j} \neq M_{j}$. There exists a nonzero homomorphism

$$
f: M_{j} /\left(L \cap M_{j}\right) \longrightarrow M_{j}
$$

Since $K \subseteq L$, there exists a natural epimorphism

$$
h: M / K \longrightarrow M / L \text {. }
$$

Hence the composition of the following homomorphisms is nonzero:

$$
\frac{M}{K} \stackrel{h}{\longrightarrow} \frac{M}{L}=\frac{M_{j}+L}{L} \cong \frac{M_{j}}{L \cap M_{j}} \stackrel{f}{\longrightarrow} M_{j} \hookrightarrow M .
$$

Therefore, $M$ is coretractable. 
In general an infinite direct sum of coretractable modules is not necessarily coretractable (see Proposition 3.2 and Example 3.3). However, under certain conditions, Proposition 2.6 holds for an infinite direct sum.

Recall that a ring $R$ is said to be right max if every nonzero right $R$-module has a maximal submodule.

PROpOSITION 2.7. Let $R$ be a right max ring and $\left\{M_{\alpha} \mid \alpha \in I\right\}$ be a family of coretractable $R$-modules. Then $M=\bigoplus_{\alpha \in I} M_{\alpha}$ is also coretractable.

PROOF. Let $K$ be a proper submodule of $M$. There exists a maximal submodule $L$ of $M$ with $K \subseteq L$. Thus for some $\beta \in I, M_{\beta} \nsubseteq L$. Now, as in the proof of Proposition 2.6, we have the desired nonzero homomorphism $M / K \longrightarrow M$.

Let $U$ and $M$ be $R$-modules. $U$ is said to be $M$-injective if for any monomorphism $f: K \longrightarrow M$ and any homomorphism $g: K \longrightarrow U$ there exists a homomorphism $h: M \longrightarrow U$ such that $h f=g$. If $M$ is $M$-injective, $M$ is called quasi-injective.

Proposition 2.8. Let $\left\{M_{\alpha} \mid \alpha \in I\right\}$ be a family of coretractable R-modules. If for any $\alpha, \beta \in I, M_{\beta}$ is $M_{\alpha}$-injective, then $M=\bigoplus_{\alpha \in I} M_{\alpha}$ is coretractable. In particular, if $M$ is a quasi-injective coretractable $R$-module, then $\bigoplus_{J} M$ is coretractable for any index set $\mathrm{J}$.

Proof. Let $K$ be a proper submodule of $M$. Then there exists $\beta \in I$ such that $M_{\beta} \nsubseteq K$. Therefore, there exists a nonzero homomorphism

$$
g: M_{\beta} /\left(K \cap M_{\beta}\right) \longrightarrow M_{\beta}
$$

Also the natural map

$$
f: M_{\beta} /\left(K \cap M_{\beta}\right) \longrightarrow M / K
$$

is a monomorphism. Since $M_{\beta}$ is $M_{\alpha}$-injective (for any $\alpha \in I$ ), $M_{\beta}$ is $(M / K)$-injective by Anderson and Fuller [2, Proposition 16.13]. So there exists $h: M / K \longrightarrow M_{\beta}$ such that $h f=g$. Hence $0 \neq i h \in \operatorname{Hom}_{R}(M / K, M)$, where $i: M_{\beta} \longrightarrow M$ is the natural inclusion.

The following example shows that an infinite direct product of coretractable modules need not be coretractable.

EXAmple 2.9. Let $P$ be the set of all prime numbers and $M=\prod_{p \in P} \mathbb{Z}_{p}$. Then $M$ is not a coretractable $\mathbb{Z}$-module. In fact, let

$$
K=\bigoplus_{p \in P} \mathbb{Z}_{p} \quad \text { and } \quad f \in \operatorname{Hom}_{\mathbb{Z}}\left(M / K, \mathbb{Z}_{p}\right)
$$

for some $p \in P$. Then

$$
M / K=(p M+K) / K=p(M / K) \subseteq \operatorname{ker}(f)
$$


and so $f=0$. Hence

$$
\operatorname{Hom}_{\mathbb{Z}}(M / K, M) \cong \prod_{p \in P} \operatorname{Hom}_{\mathbb{Z}}\left(M / K, \mathbb{Z}_{p}\right)=0,
$$

as desired.

Let $M$ be an $R$-module. A submodule $K$ is said to be dense in $M$ if, for any $y \in M$ and $0 \neq x \in M$, there exists $r \in R$ such that $x r \neq 0$ and $y r \in K$. Obviously, any dense submodule of $M$ is essential. The following result is in Lam [8, Proposition 8.6].

LEMMA 2.10. Let $M$ be an $R$-module and $K \leq M$. Then the following statements are equivalent:

(a) $K$ is a dense submodule of $M$;

(b) $\operatorname{Hom}_{R}(M / K, \mathrm{E}(M))=0$;

(c) $\operatorname{Hom}_{R}(P / K, M)=0$ for any submodule $P$ with $K \subseteq P \subseteq M$.

COROLlary 2.11. Let $M$ be a coretractable R-module. Then $M$ has no proper dense submodules.

The following example shows that the converse of Corollary 2.11 is not true.

ExAmple 2.12. Let $M$ be as in Example 2.9. Then $M$ is not a coretractable $\mathbb{Z}$-module. However, we show that $M$ has no proper dense submodules. Let $K \unlhd M$ and $y \in M \backslash K$. There exists an $n>1$ such that

$$
\{r \in \mathbb{Z} \mid y r \in K\}=n \mathbb{Z} .
$$

There also exists $0 \neq x \in M$ such that $x n=0$. Thus for any $r \in \mathbb{Z}$ with $y r \in K, x r=0$ and hence $K$ is not dense in $M$, as required.

The following result gives a necessary and sufficient condition for a quasi-injective or finitely generated module to be coretractable.

THEOREM 2.13. Let $M$ be an R-module which satisfies one of the following conditions:

(a) $M$ is quasi-injective;

(b) every proper submodule of $M$ is contained in a maximal submodule.

Then $M$ is coretractable if and only if it has no proper dense submodules.

PROOF. The necessity is clear by Corollary 2.11 .

(a) Let $K$ be a proper submodule of $M$. Since $K$ is not dense in $M$, by Lemma 2.10, $\operatorname{Hom}_{R}(P / K, M) \neq 0$ for some submodule $K \subseteq P \subseteq M$. So there is a nonzero homomorphism $g: P \longrightarrow M$ with $g(K)=0$. We can extend $g$ to $h: M \longrightarrow M$ by hypothesis and hence $\operatorname{Hom}_{R}(M / K, M) \neq 0$.

(b) Let $K$ be a proper submodule of $M$. There is a maximal submodule $L$ of $M$ with $K \subseteq L$. Since $L$ is a maximal submodule of $M$ which is not dense in $M$, again by Lemma 2.10, $\operatorname{Hom}_{R}(M / L, M) \neq 0$ and hence $\operatorname{Hom}_{R}(M / K, M) \neq 0$. 
A ring $R$ is said to be right Kasch if every simple right $R$-module can be embedded into $R_{R}$ (see, for example, Lam [8, p. 280]).

THEOREM 2.14. For a ring $R$, the following statements are equivalent:

(a) $R$ is a right Kasch ring;

(b) $R_{R}$ is a coretractable module;

(c) every finitely generated free right $R$-module is coretractable;

(d) for any right ideal $I$ of $R, l_{R}(I) \neq 0$;

(e) $R_{R}$ has no proper dense submodules.

Proof. By Proposition 2.6, statements (b) and (c) are equivalent, and by Theorem 2.14, (b) and (e) are equivalent. The equivalence of (b) and (d) follows from the fact that any endomorphism of $R_{R}$ is given by left multiplication by some element of $R$. Finally, since every proper right ideal of $R$ is contained in a maximal right ideal, one can easily see that (a) and (b) are equivalent.

Example 2.15 shows that being coretractable is not preserved by extensions.

EXAmple 2.15. Let $K$ be a field and let $R$ be the ring of $2 \times 2$ upper triangular matrices over $K$. Then $J=\left(\begin{array}{ll}0 & K \\ 0 & 0\end{array}\right)$ is the Jacobson radical of $R$. Since $J$ and $R / J$ are semisimple, they are both coretractable but $R$ is not so (for $I=\left(\begin{array}{ll}0 & K \\ 0 & K\end{array}\right)$ we have $l_{R}(I)=0$ and now apply Theorem 2.14).

REMARK 2.16. Let $M$ be a nonzero $R$-module. An $R$-module $N$ is subgenerated by $M$ if $N$ is isomorphic to a submodule of an $M$-generated module. The full subcategory of Mod- $R$ consisting of all $R$-modules that are subgenerated by $M$ is denoted by $\sigma[M]$ (see Wisbauer [12, p. 118]). Following Albu and Wisbauer [1], an $R$-module $M$ is called a Kasch module if it contains a copy of every simple module in $\sigma[M]$. So a ring $R$ is a right Kasch ring if and only if $R_{R}$ is a Kasch module. Consequently, by Theorem 2.14, $R_{R}$ is coretractable if and only if it is a Kasch module. In the following example we show that for an $R$-module $M$ these are two different concepts.

EXAmple 2.17. (a) Let $R=\mathbb{Z}$ and let $M$ be as in Example 2.9. Since all simple $R$-modules can be embedded into $M, M$ is a Kasch module which is not coretractable. However, a Kasch module which is finitely generated or quasi-injective is coretractable.

(b) Let $K$ be a field and let $R$ be the ring of all matrices of the form

$$
r=\left(\begin{array}{ccc}
a & x & y \\
0 & b & z \\
0 & 0 & a
\end{array}\right) \quad \text { where } a, b, x, y, z \in K .
$$

Let

$$
M=\{r \in R \mid b=z=0\} .
$$

Then $M$ is a right $R$-module with exactly two proper nonzero submodules $A$ and $B$, where

$$
A=\{r \in R \mid a=b=z=0\}
$$


and

$$
B=\{r \in R \mid a=b=x=z=0\} .
$$

Also $M / A \cong B=\operatorname{Soc}\left(M_{R}\right)$ and hence $M_{R}$ is coretractable. Since $A / B \in \sigma[M]$ is a simple $R$-module and $A / B \mp B$, we deduce that $M$ is not a Kasch module, as required.

\section{Classes of coretractable modules}

The aim of this section is to find conditions under which some classes of modules are coretractable.

PROPOSITION 3.1. For a ring $R$, the following statements are equivalent:

(a) all free $R$-modules are coretractable:

(b) $\operatorname{Hom}_{R}(M, R) \neq 0$ for every nonzero $R$-module $M$;

(c) $\operatorname{Hom}_{R}(M, R) \neq 0$ for every nonzero singular $R$-module $M$.

PRoOF. To show that (a) implies (b), let $M$ be a nonzero $R$-module. There is a free $R$-module $F$ and $K \leq F$ such that $M \cong F / K$. Since $F$ is coretractable, $\operatorname{Hom}_{R}(F / K, F) \neq 0$ and so $\operatorname{Hom}_{R}(M, R) \cong \operatorname{Hom}_{R}(F / K, R) \neq 0$.

Clearly (b) implies (c).

Finally, to prove that (c) implies (a), let $F$ be a free $R$-module and $K$ be a proper essential submodule of $F$. Since $F / K$ is a (nonzero) singular $R$-module, $\operatorname{Hom}_{R}(F / K, R) \neq 0$ and so $\operatorname{Hom}_{R}(F / K, F) \neq 0$. Therefore, $F$ is coretractable.

Note that by Proposition 2.7, over a right max ring all free $R$-modules are coretractable if $R$ is a right Kasch ring. But by the next result we see that the condition ' $R$ is a right max ring' cannot be omitted from the assertion.

PROPOSITION 3.2. Let $R$ be a right Kasch ring which is not right max. If every right ideal of $R$ has a maximal submodule, then there is a free $R$-module which is not coretractable.

PROOF. There is an $R$-module $M$ with no maximal submodules. Since every right ideal of $R$ has a maximal submodule, $\operatorname{Hom}_{R}(M, R)=0$. Let $M \cong F / K$, where $F$ is a free $R$-module and $K \leq F$. Then $\operatorname{Hom}_{R}(F / K, F)=0$ and so $F$ is not coretractable.

The following example guarantees the existence of a ring with the required conditions of Proposition 3.2. Recall that a subset $X$ of a ring $R$ is said to be right (left) $T$-nilpotent in case for every sequence $a_{1}, a_{2}, \ldots$ in $X$, there is an integer $n \geq 1$ such that $a_{n} a_{n-1} \cdots a_{1}=0\left(a_{1} a_{2} \cdots a_{n}=0\right)$. It is well known that for a right max ring $R, \mathrm{~J}(R)$ is right $T$-nilpotent.

EXAMPLE 3.3. Let $R$ be the ring of all $\mathbb{N} \times \mathbb{N}$ lower triangular matrices over a field $K$ which are constant on the diagonal and have only finitely many nonzero entries off the diagonal. Then $R$ is a local ring and $J=\mathrm{J}(R)$ is the set of all matrices with zero diagonal. It is easy to check that $J$ is not right $T$-nilpotent and so $R$ is not a right 
max ring. Let $0 \neq x \in R$ have nonzero entries only in the first column. Then $x J=0$ (and thus $x R \cong R / J$ ) which implies that $R$ is a right Kasch ring. It is not difficult to see that $\bigcap_{n=1}^{\infty} J^{n}=0$. So for any nonzero proper right ideal $I$ of $R$ there exists $n \geq 1$ such that $I \subseteq J^{n}$ and $I \nsubseteq J^{n+1}$. Since

$$
I /\left(I \cap J^{n+1}\right) \cong\left(I+J^{n+1}\right) / J^{n+1} \leq J^{n} / J^{n+1},
$$

$I /\left(I \cap J^{n+1}\right)$ is an $(R / J)$-module and so has a maximal submodule. Therefore, $I$ has a maximal submodule, as desired.

We now consider rings over which every module is coretractable. But for convenience we give the following definition.

DEFINITION 3.4. Let $R$ be a ring. We call $R$ right completely coretractable (right CC) if every right $R$-module is coretractable. Left completely coretractable (left CC) rings are defined similarly. A ring is said to be completely coretractable (CC) if it is both left and right $\mathrm{CC}$.

Recall that a ring $R$ is right semi-Artinian if every right $R$-module has a minimal submodule (equivalently, if every cyclic right $R$-module has a minimal submodule; see Stenstrom [10, Proposition VIII.2.5]).

PROPOSITION 3.5. Let $R$ be a right semi-Artinian right max ring. If $R$ has a unique simple right $R$-module (up to isomorphism), then $R$ is a right $C C$ ring.

PROOF. Let $M$ be a nonzero $R$-module and $K$ be a proper submodule of $M$. There is a maximal submodule $L$ of $M$ with $K \subseteq L$. Since $R$ is right semi-Artinian with a unique simple (right) $R$-module, $M / L$ can be embedded into $M$ and so $\operatorname{Hom}_{R}(M / K, M) \neq 0$. Therefore, $M$ is coretractable.

Note that a semisimple ring is (right) CC although it does not have necessarily a unique simple module.

PROpOSITION 3.6. Let $R$ be a right max ring. If every cyclic $R$-module is coretractable, then $R$ is a right $C C$ ring.

PROOF. Let $K$ be a proper submodule of an $R$-module $M$. There is a maximal submodule $L$ of $M$ containing $K$. Let $x \in M \backslash L$; then $M / L \cong x R /(L \cap x R)$. Thus $\operatorname{Hom}_{R}(M / L, x R) \neq 0$ which implies that $\operatorname{Hom}_{R}(M / K, M) \neq 0$. Therefore, $M$ is coretractable.

Let $R$ be the ring of Example 3.3. Since $R$ is a local left perfect ring, it is right semiArtinian with a unique simple $R$-module. Then as in the proof of Proposition 3.5, we can show that all finitely generated $R$-modules are coretractable. However, by Proposition 3.2, $R$ is not a right $\mathrm{CC}$ ring.

We now investigate some properties of (right) CC rings. But first we need a lemma.

LEMMA 3.7. Let $R$ be a right Kasch ring. If $\mathrm{J}(R)=0$, then $R$ is semisimple. 
Proof. Let $T=\operatorname{Soc}\left(R_{R}\right)$. If $T \neq R$, then since $R$ is a right Kasch ring, by Theorem 2.14, $I=l_{R}(T) \neq 0$. Thus $(T I)^{2}=0$. As $\mathrm{J}(R)=0$, we have $T I=0$. Since $R$ is right Kasch, $T$ contains a copy of every simple right $R$-module and so $I$ annihilates all simple right $R$-modules. Therefore, $I \subseteq \mathrm{J}(R)=0$ which is a contradiction. Thus $T=R$ and hence $R$ is semisimple.

PROPOSITION 3.8. Let $R$ be a ring. If all cyclic right $R$-modules are coretractable, then $R$ is a left perfect ring.

Proof. Let $J=\mathrm{J}(R)$. Since $R / J$ is coretractable as an $R$-module (and hence as an $(R / J)$-module), it is a right Kasch ring. So by Lemma 3.7, $R / J$ is a semisimple ring. Now let $M$ be a nonzero cyclic right $R$-module. Since $\operatorname{Rad}(M) \neq M$, by Proposition 2.3, $\operatorname{Soc}(M) \neq 0$ and hence $R$ is right semi-Artinian. Therefore, $J$ is left $T$-nilpotent (see, for example, Stenstrom [10, Corollary VIII.2.7]) and so $R$ is a left perfect ring.

Let $R$ be a ring and $I$ be a nonzero two-sided ideal of $R$. Set $I^{0}=R$ and for any ordinal $\alpha>0$ we define $I^{\alpha}=I^{\beta} I$ if $\alpha=\beta+1$ for some ordinal $\beta$ and $I^{\alpha}=\bigcap_{0 \leq \beta<\alpha} I^{\beta}$ if $\alpha$ is a limit ordinal. So $\left\{I^{\alpha} \mid \alpha\right.$ is an ordinal $\}$ is a descending chain of ideals of $R$. There is an ordinal $\lambda$ such that $I^{\lambda}=I^{\gamma}$ for any ordinal $\gamma \geq \lambda$. Now let $\kappa(I)=I^{\lambda}$.

LEMMA 3.9. Let $R$ be a right $C C$ ring and let $J=\mathrm{J}(R)$. If $\kappa(J)=0$, then $R$ is a right max ring.

Proof. By Proposition 3.1, $\operatorname{Hom}_{R}(M, R) \neq 0$ for any nonzero right $R$-module $M$. So to prove that $R$ is a right max ring, it suffices to show that any nonzero right ideal of $R$ has a maximal submodule. Let $I$ be a right ideal of $R$ which has no maximal submodules. By Proposition $3.8, R / J$ is semisimple and so Corollary 15.18 of Anderson and Fuller [2] implies that $I=\operatorname{Rad}(I)=I J \subseteq J$. It is easy to show that $I \subseteq \kappa(J)$ and hence $I=0$, as required.

THEOREM 3.10. Let $R$ be a right $C C$ ring and let $J=\mathrm{J}(R)$. Then $\kappa(J)=0$ and so $R$ is a (two-sided) perfect ring.

PROOF. Suppose on the contrary that $L=\kappa(J) \neq 0$. By Proposition 3.8, $J$ is left $T$-nilpotent and so $L^{2} \subseteq J L \varsubsetneqq L$ (see Anderson and Fuller [2, Lemma 28.3]). Let $T=R / L$. Then by Lemma 3.13, $T$ is a right CC ring with the Jacobson radical $\mathrm{J}(T)=J / L$. Since $\kappa(\mathrm{J}(T))=0$, by Lemma 3.9, $T$ is a right max ring. Now $L / L^{2}$ is a nonzero right $T$-module and hence has a maximal submodule. As a consequence $L_{R}$ also has a maximal submodule and so $L J=\operatorname{Rad}\left(L_{R}\right) \neq L$. This is a contradiction because $\kappa(I) I=\kappa(I)$ for any ideal $I$ of $R$. Therefore, $\kappa(J)=0$ and by Lemma 3.9, $R$ is a right max ring. Thus $J$ is right $T$-nilpotent. Since $R$ is a left perfect ring, it is also right perfect. 
Corollary 3.11. Let $R$ be a right $C C$ ring. Then a right (left) $R$-module is Noetherian if and only if it is Artinian.

PROOF. Since $R$ is a (two-sided) perfect ring, it is a (two-sided) semi-Artinian max ring. However, over a semi-Artinian max ring a module is Noetherian if and only if it is Artinian (see Stenstrom [10, Proposition VIII.2.1]).

COROllary 3.12. Let $R$ be a right semi-Artinian max ring with a unique simple right $R$-module. Then $R$ is a (two-sided) perfect ring. $R$ is also a left $C C$ ring.

Proof. By Proposition 3.5, $R$ is a right $\mathrm{CC}$ ring and so it is (two-sided) perfect. Let $J=\mathrm{J}(R)$. Since $R$ has a unique simple right $R$-module, $R / J$ is a simple Artinian ring and hence $R$ has also a unique simple left $R$-module. As $R$ is a left semi-Artinian max ring, again by Proposition 3.5, $R$ is a left CC ring.

The converse of Theorem 3.10 holds for commutative rings. First we need a lemma.

LEMMA 3.13. Let $R$ and $S$ be rings and let $I$ be an ideal of $R$.

(a) If $R$ is a right $C C$ ring, then so is $R / I$.

(b) We have $R \times S$ is right $C C$ if and only if $R$ and $S$ are both right $C C$.

PROOF. (a) Follows from the fact that every $(R / I)$-module is obviously an $R$-module.

(b) The necessity is clear by part (a). For the sufficiency note that every $(R \times S)$ module is a direct sum of an $R$-module and an $S$-module.

THEOREM 3.14. Let $R$ be a commutative ring. Then the following statements are equivalent:

(a) $R$ is a $C C$ ring;

(b) every cyclic $R$-module is coretractable;

(c) $R$ is a perfect ring.

Proof. Clearly (a) implies (b) and, by Proposition 3.8, (b) implies (c). Now suppose that (c) holds. By Lam [7, Theorem 23.24], $R=R_{1} \times \cdots \times R_{n}$ where each $R_{i}$ is a local perfect ring $(1 \leq i \leq n)$. Since a commutative local perfect ring is a semiArtinian max ring with a unique simple module, by Proposition 3.5, each $R_{i}$ is a CC ring. Now Lemma 3.13 implies that $R$ is a CC ring and therefore (a) is satisfied.

Note that Theorem 3.14 is not valid for noncommutative rings, as is illustrated in the following example.

EXAMPLE 3.15. Let $K$ be a division ring and let $R$ be the ring of all $4 \times 4$ matrices of the form

$$
\left(\begin{array}{llll}
a & x & 0 & 0 \\
0 & b & 0 & 0 \\
0 & 0 & b & y \\
0 & 0 & 0 & a
\end{array}\right)
$$


where $a, b, x, y \in K$. Then $R$ is a quasi-Frobenius (and hence two-sided perfect and Kasch) ring and $J=\mathrm{J}(R)$ consists of all matrices in $R$ with zero diagonal. Let

$$
e=\left(\begin{array}{llll}
1 & 0 & 0 & 0 \\
0 & 0 & 0 & 0 \\
0 & 0 & 0 & 0 \\
0 & 0 & 0 & 1
\end{array}\right) \in R
$$

Then $\operatorname{Soc}(e R)=e J$ is a simple $R$-module. Also $e R / e J$ is a simple $R$-module which is not isomorphic to $e J$. Therefore, $\operatorname{Hom}_{R}(e R / e J, e R)=0$ and so $e R$ is not coretractable. In particular, $R$ is not a right $\mathrm{CC}$ ring. For the details see Lam [8, Example 16.19(4)].

Note that in Example 3.15 since $R$ is a Kasch and max ring, all free $R$-modules are coretractable. But the projective module $e R$ is not coretractable.

\section{Endomorphism ring of coretractable modules}

Throughout this section, $M_{R}$ is a right $R$-module and $S=\operatorname{End}_{R}(M)$ is the ring of $R$-endomorphisms of $M$. Hence ${ }_{S} M_{R}$ is a bimodule.

We begin with some characterizations of coretractable modules in terms of their endomorphism rings.

LEMMA 4.1. For a right $R$-module $M$, the following statements are equivalent:

(a) $M_{R}$ is coretractable;

(b) for any proper submodule $K$ of $M_{R}, r_{M}\left(l_{S}(K)\right) / K \ll M / K$;

(c) for any submodule $K$ of $M_{R}$, if $r_{M}\left(l_{S}(K)\right)$ is a summand of $M_{R}$, then $K=r_{M}\left(l_{S}(K)\right)$.

PROOF. To prove that (a) implies (b), let $K$ be a proper submodule of $M_{R}$ and $L=r_{M}\left(l_{S}(K)\right)$. Suppose that

$$
(L / K)+(U / K)=M / K
$$

If $U \neq M$, then by hypothesis, there exists $0 \neq f \in S$ with $f(U)=0$. Since $K \subseteq U$, $f \in l_{S}(K)$ and so $f(L)=0$. Hence $f(M)=f(L+U)=0$, which is a contradiction. Therefore, $L / K \ll M / K$.

We now show that (b) implies (c). Let $K$ be a proper submodule of $M_{R}$ and let $L=r_{M}\left(l_{S}(K)\right)$ be a summand of $M_{R}$. So $M=L \oplus U$ for some $U \leq M_{R}$. Thus

$$
(L / K)+((U+K) / K)=M / K \text {. }
$$

Since $L / K \ll M / K, U+K=M$ which implies that $K=L$, as desired.

Finally, to check that (c) implies (a), let $K \leq M_{R}$ and $\operatorname{Hom}_{R}(M / K, M)=0$. Then $l_{S}(K)=0$ and so $r_{M}\left(l_{S}(K)\right)=M$ is a summand of $M$. Therefore, $K=M$ and hence $M_{R}$ is coretractable. 
Recall that an $R$-module $M_{R}$ is co-semisimple if for any $K \leq M_{R}, \operatorname{Rad}(M / K)=0$ (see Wisbauer [12, p. 190]).

COROLlary 4.2. Let $M_{R}$ be a coretractable module. Then:

(a) for any $K \ll M_{R}$, we have $r_{M}\left(l_{S}(K)\right) \ll M_{R}$;

(b) if $K=\operatorname{Rad}\left(M_{R}\right)$ or $K$ is a maximal submodule of $M_{R}$, then $r_{M}\left(l_{s}(K)\right)=K$ (that is, $M$ cogenerates $M / K$ );

(c) if $M_{R}$ is co-semisimple, then $r_{M}\left(l_{S}(K)\right)=K$ for any $K \leq M_{R}$.

Proof. (a) This follows from the fact that for $K \leq L \leq M$ if $K \ll M$ and $L / K \ll$ $M / K$, then $L \ll M$.

(b) and (c) In both cases, since $\operatorname{Rad}(M / K)=0, M / K$ has no nonzero superfluous submodules.

The following result shows that when the endomorphism ring of a coretractable module is coretractable.

PROposition 4.3. Let $M_{R}$ be coretractable. Then $S_{S}$ is coretractable if and only if $I M \neq M$ for any proper right ideal I of $S$.

PROOF. In general, if $S_{S}$ is coretractable, then by Theorem 2.14 , for any proper right ideal $I$ of $S$, there exists $0 \neq f \in S$ with $f I=0$. Thus $f(I M)=0$, which implies that $I M \neq M$. Conversely, let $I$ be a proper right ideal of $S$. Since $I M \neq M$, there exists $0 \neq f \in S$ with $f(I M)=0$. Therefore, $f I=0$ and, again by Theorem $2.14, S_{S}$ is coretractable.

Note that the endomorphism ring of a coretractable module need not be coretractable. For example, let $R=\mathbb{Z}$ and $M=\mathbb{Z}_{p^{\infty}}$. Then $S=\operatorname{End}_{R}(M)$ is a domain which is not a coretractable $S$-module by Theorem 2.14 .

We know that the endomorphism ring of a semisimple module is a regular ring. For coretractable modules the converse does also hold.

Proposition 4.4. Let $M_{R}$ be coretractable. If $S$ is a regular ring, then $M_{R}$ is semisimple.

Proof. Suppose on the contrary that $M_{R}$ is not semisimple. So $M_{R}$ has a proper essential submodule $K$. Then $f(K)=0$ for some $0 \neq f \in S$. There exists $g \in S$ such that $f=f g f$. Since $(g f)^{2}=g f$ and $\operatorname{ker}(f)=\operatorname{ker}(g f), \operatorname{ker}(f)$ is a summand of $M_{R}$ which contains the essential submodule $K$, and this is a contradiction.

Following Wisbauer [12, p. 261], an $R$-module $M_{R}$ is called semi-injective if for any $f \in S$,

$$
S f=l_{S}(\operatorname{ker}(f))=l_{S}\left(r_{M}(f)\right)
$$

(equivalently, for any monomorphism $f: N \longrightarrow M$, where $N$ is a factor module of $M_{R}$, and for any homomorphism $g: N \longrightarrow M$, there exists $h: M \longrightarrow M$ such that $h f=g$ ). Clearly every quasi-injective module is semi-injective. Also for a ring $R, R_{R}$ is semi-injective if and only if it is right principally injective. In the 
following proposition, we establish relations between submodules of a coretractable module and its endomorphism ring.

Proposition 4.5. Let $M_{R}$ be a coretractable module and let $K \leq M_{R}$ and $I \leq{ }_{S} S$.

(a) $\quad$ If $l_{S}(K) \unlhd{ }_{S} S$, then $K \ll M_{R}$.

(b) If $I \unlhd{ }_{S} S$, then $r_{M}(I) \ll M_{R}$.

Moreover, suppose that $M_{R}$ is semi-injective.

(c) If $K \ll M_{R}$, then $l_{S}(K) \unlhd{ }_{S} S$.

(d) If $K$ is a maximal submodule of $M_{R}$, then $l_{S}(K)$ is a minimal left ideal of $S$.

(e) If I is a minimal left ideal of $S$, then $r_{M}(I)$ is a maximal submodule of $M_{R}$.

PROOF. (a) Let $L$ be a proper submodule of $M_{R}$. There exists $0 \neq f \in S$ with $f(L)=0$. Since $l_{S}(K) \unlhd{ }_{S} S$, there exists $g \in S$ such that $0 \neq g f \in l_{S}(K)$. Therefore, $g f(K+L)=0$ and hence $K+L \neq M$. Consequently, $K \ll M_{R}$.

(b) This follows from part (a) and the fact that $I \subseteq l_{S}\left(r_{M}(I)\right)$.

(c) Suppose that $S f \cap l_{S}(K)=0$ for some $f \in S$. Since $M_{R}$ is semi-injective,

$$
0=S f \cap l_{S}(K)=l_{S}(\operatorname{ker}(f)) \cap l_{S}(K)=l_{S}(\operatorname{ker}(f)+K) .
$$

But $M_{R}$ is coretractable and so $\operatorname{ker}(f)+K=M$. Therefore, $\operatorname{ker}(f)=M$ which implies that $f=0$. Hence $l_{S}(K) \unlhd{ }_{S} S$.

(d) As $M$ is coretractable, $l_{S}(K) \neq 0$. Let $0 \neq f \in l_{S}(K)$. Since $f(K)=0$ and $K$ is a maximal submodule of $M_{R}, \operatorname{ker}(f)=K$. Thus $l_{S}(K)=l_{S}(\operatorname{ker}(f))=S f$.

(e) Suppose that $r_{M}(I) \subseteq K$ for a proper submodule $K$ of $M_{R}$. So

$$
0 \neq l_{S}(K) \subseteq l_{S}\left(r_{M}(I)\right) .
$$

Since $M_{R}$ is semi-injective and $I$ is a cyclic left ideal of $S, I=l_{S}\left(r_{M}(I)\right)$. Thus $l_{S}(K)=I$ and so $K \subseteq r_{M}\left(l_{S}(K)\right)=r_{M}(I)$. Therefore, $r_{M}(I)$ is a maximal submodule of $M_{R}$.

A nonzero module is said to be uniform if each nonzero submodule is essential. It is said to be hollow (co-uniform) if each proper submodule is superfluous.

COROLlaRY 4.6. Let $M_{R}$ be a nonzero coretractable module. If ${ }_{S} S$ is uniform, then $M_{R}$ is hollow. The converse holds when $M_{R}$ is semi-injective.

Proof. Suppose that ${ }_{S} S$ is uniform. Let $K$ be a proper submodule of $M_{R}$. Since $l_{S}(K) \neq 0$, it is an essential left ideal of $S$. Now Proposition 4.5(a) implies that $K \ll M_{R}$. Therefore, $M_{R}$ is hollow.

Now suppose that $M_{R}$ is semi-injective and hollow. Let $0 \neq f \in S$. Since $S f=$ $l_{S}\left(r_{M}(f)\right)$ and $r_{M}(f) \ll M_{R}$, by Proposition 4.5(c), $S f \unlhd{ }_{S} S$. Consequently, ${ }_{S} S$ is uniform.

Let $R$ be the ring of Example 3.3. Since $R$ is a local right Kasch ring, $R_{R}$ is coretractable and hollow. But $R=\operatorname{End}_{R}(R)$ is not a left uniform $R$-module. Thus, the semi-injectivity condition is needed in the last statement of Corollary 4.6. 
COROllary 4.7. Let $M_{R}$ be a semi-injective coretractable module. Then $\operatorname{Rad}\left(M_{R}\right)=r_{M}\left(\operatorname{Soc}\left({ }_{S} S\right)\right)$. In particular, $\operatorname{Rad}\left(M_{R}\right)=M$ if and only if $\operatorname{Soc}\left({ }_{S} S\right)=0$.

PRoOF. We have

$$
l_{S}\left(\operatorname{Rad}\left(M_{R}\right)\right)=l_{S}\left(\sum_{K \ll M_{R}} K\right)=\bigcap_{K \ll M_{R}} l_{S}(K) .
$$

By Proposition 4.5(c), $l_{S}(K) \unlhd{ }_{S} S$ for any $K \ll M_{R}$. Thus $\operatorname{Soc}\left({ }_{S} S\right) \subseteq l_{S}\left(\operatorname{Rad}\left(M_{R}\right)\right)$, equivalently, $\operatorname{Rad}\left(M_{R}\right) \subseteq r_{M}\left(\operatorname{Soc}\left({ }_{S} S\right)\right)$. Now suppose that $x \in M \backslash \operatorname{Rad}\left(M_{R}\right)$. There is a maximal submodule $K$ of $M_{R}$ with $x \notin K$. Let $0 \neq f \in l_{S}(K)$. By Proposition $4.5(\mathrm{~d}), l_{S}(K)$ is a minimal left ideal of $S$ and so $f \in \operatorname{Soc}\left({ }_{S} S\right)$. Since $f(x) \neq 0, x \notin r_{M}(\operatorname{Soc}(S S))$. Therefore, $\operatorname{Rad}\left(M_{R}\right)=r_{M}\left(\operatorname{Soc}\left({ }_{S} S\right)\right)$. Now the last assertion follows from this equality.

As a consequence of Corollary 4.7, we see that for a semi-injective coretractable module $M_{R}$, if $\operatorname{Soc}\left({ }_{S} S\right)$ is a summand of $S S$, then $\operatorname{Rad}\left(M_{R}\right)$ is a summand of $M_{R}$.

COROLlary 4.8. Let $M$ be a coretractable R-module. Then

$$
Z\left({ }_{S} S\right) \subseteq\left\{f \in S \mid f(M) \ll M_{R}\right\} .
$$

The equality holds when $M_{R}$ is semi-injective.

PRoOF. Let $f \in Z\left({ }_{S} S\right)$. There is an essential left ideal $I$ of $S$ such that $I f=0$. Thus $f(M) \subseteq r_{M}(I)$ and by Proposition 4.5(b), $r_{M}(I) \ll M_{R}$. Therefore, $f(M) \ll M_{R}$. Now suppose that $M_{R}$ is semi-injective and $f \in S$ with $f(M) \ll M_{R}$. Then again by Proposition 4.5(c),

$$
l_{S}(f)=l_{S}(f(M)) \unlhd{ }_{S} S .
$$

Therefore, $f \in Z\left({ }_{S} S\right)$.

Observe that by Corollary 4.8, a coretractable module with zero Jacobson radical has a left nonsingular endomorphism ring.

Let $M$ be a nonzero $R$-module. The uniform dimension (or Goldie dimension) of $M$ is defined as

$$
\text { u.dim }\left(M_{R}\right)=\sup \{k \mid M \text { contains a direct sum of } k \text { nonzero submodules }\} .
$$

There are various dual notions for the uniform dimension (see, for example, [3, 11]). Varadarajan [11] defined the corank of a nonzero module $M$ as

$$
\operatorname{corank}(M)=\sup \left\{k \mid \text { there is an epimorphism } M \longrightarrow \prod_{i=1}^{k} N_{i} \text { with all } N_{i} \neq 0\right\} .
$$

We now investigate the relation between $\operatorname{corank}\left(M_{R}\right)$ and $\operatorname{uddim}\left({ }_{S} S\right)$ for a coretractable module $M_{R}$. But first we need a lemma. 
Lemma 4.9. Let $M_{R}$ be a nonzero $R$-module and $K, L \leq M_{R}$ with $K+L=M$. Then $l_{S}(K \cap L)=l_{S}(K)+l_{S}(L)$.

Proof. Clearly $l_{S}(K)+l_{S}(L) \subseteq l_{S}(K \cap L)$. Now let $f \in l_{S}(K \cap L)$. Define $g, h \in$ $S$ as follows. For any $m \in M$, let $g(m)=f(x)$ and $h(m)=f(y)$ if $m=x+y$, where $x \in K$ and $y \in L$. Since $f(K \cap L)=0, g$ and $h$ are well defined. Obviously, $f=g+h$ and $g \in l_{S}(L)$ and $h \in l_{S}(K)$, as desired.

PROpOSITION 4.10. Let $M_{R}$ be a nonzero coretractable module. Then $\mathrm{u} \cdot \operatorname{dim}\left({ }_{S} S\right) \geq$ $\operatorname{corank}\left(M_{R}\right)$.

PROOF. Suppose that $f: M \longrightarrow N_{1} \times \cdots \times N_{n}$ is an epimorphism, where each $N_{i} \neq 0(1 \leq i \leq n)$. Let $\pi_{i}: N_{1} \times \cdots \times N_{n} \longrightarrow N_{i}$ be the natural projection and let $K_{i}=\operatorname{ker}\left(\pi_{i} f\right)$ for all $1 \leq i \leq n$. For each $1 \leq i \leq n$, put $L_{i}=\bigcap_{j \neq i} K_{j}$. Since $f$ is an epimorphism, $K_{i}+L_{i}=M(1 \leq i \leq n)$. If $I_{i}=l_{S}\left(K_{i}\right)$, then by induction and using Lemma 4.9 , it is easy to check that $l_{S}\left(L_{i}\right)=\sum_{j \neq i} l_{S}\left(K_{j}\right)=\sum_{j \neq i} I_{j}$. Since $M_{R}$ is coretractable, $I_{i} \neq 0$ for each $i$ and

$$
0=l_{S}(M)=l_{S}\left(K_{i}+L_{i}\right)=l_{S}\left(K_{i}\right) \cap l_{S}\left(L_{i}\right)=I_{i} \cap \sum_{j \neq i} I_{j} .
$$

Therefore, $\left\{I_{1}, \ldots, I_{n}\right\}$ is an independent set of nonzero left ideals of $S$. Thus $\mathrm{u} \cdot \operatorname{dim}\left({ }_{S} S\right) \geq n$ and hence, by definition, $\mathrm{u} \cdot \operatorname{dim}\left({ }_{S} S\right) \geq \operatorname{corank}\left(M_{R}\right)$.

PROPOSITION 4.11. Let $M_{R}$ be a nonzero semi-injective coretractable module. Then $\operatorname{corank}\left(M_{R}\right)=\mathrm{u} \cdot \operatorname{dim}\left({ }_{S} S\right)$.

ProOF. Suppose that $0 \neq f_{i} \in S$ for $1 \leq i \leq n$ and $\left\{S f_{1}, S f_{2}, \ldots, S f_{n}\right\}$ is an independent set of left ideals of $S$. Let $K_{i}=\operatorname{ker}\left(f_{i}\right)(1 \leq i \leq n)$, then by semiinjectivity of $M_{R}, S f_{i}=l_{S}\left(K_{i}\right)$. Thus for any $i \neq j$,

$$
0=S f_{i} \cap S f_{j}=l_{S}\left(K_{i}\right) \cap l_{S}\left(K_{j}\right)=l_{S}\left(K_{i}+K_{j}\right) .
$$

Since $M_{R}$ is coretractable, $K_{i}+K_{j}=M$. By Lemma 4.9,

$$
l_{S}\left(K_{i} \cap K_{j}\right)=l_{S}\left(K_{i}\right)+l_{S}\left(K_{j}\right) \text { for any } i \neq j .
$$

Now let $i, j, k$ be three distinct positive integers less than $n+1$. Then

$$
\begin{aligned}
l_{S}\left(K_{i}+\left(K_{j} \cap K_{k}\right)\right) & =l_{S}\left(K_{i}\right) \cap l_{S}\left(K_{j} \cap K_{k}\right) \\
& =l_{S}\left(K_{i}\right) \cap\left(l_{S}\left(K_{j}\right)+l_{S}\left(K_{k}\right)\right) \\
& =S f_{i} \cap\left(S f_{j}+S f_{k}\right)=0 .
\end{aligned}
$$

Therefore, $K_{i}+\left(K_{j} \cap K_{k}\right)=M$. Now we can show by induction that for any $1 \leq i \leq n, K_{i}+\left(\bigcap_{j \neq i} K_{j}\right)=M$. Hence the natural map

$$
\varphi: M \longrightarrow\left(M / K_{1}\right) \times \cdots \times\left(M / K_{n}\right)
$$

is an epimorphism. Consequently, $\operatorname{corank}\left(M_{R}\right) \geq \mathrm{u} \cdot \operatorname{dim}\left({ }_{S} S\right)$ and by Proposition 4.10, the equality holds. 


\section{Acknowledgements}

The authors would like to thank the referee for a careful reading of the manuscript. They would also like to express their gratitude to Professor Wisbauer for providing a copy of the paper 'Kasch modules'.

\section{References}

[1] T. Albu and R. Wisbauer, 'Kasch modules', in: Advances in Ring Theory (eds. S. K. Jain and S. T. Rizvi) (Birkhäuser, Basel, 1997), pp. 1-16.

[2] F. W. Anderson and K. R. Fuller, Rings and Categories of Modules (Springer, New York, 1992).

[3] P. Fleury, 'A note on dualizing Goldie dimension', Canad. Math. Bull. 17 (1974), 511-517.

[4] A. Haghany and M. R. Vedadi, 'Study of semi-projective retractable modules', Algebra Colloq. 14 (2007), 489-496.

[5] S. M. Khuri, 'Endomorphism rings and lattice isomorphisms', J. Algebra 56 (1979), 401-408.

[6] - 'Correspondence theorems for modules and their endomorphism rings', J. Algebra 122 (1989), 380-396.

[7] T. Y. Lam, A First Course in Noncommutative Rings (Springer, New York, 1991).

[8] Lectures on Modules and Rings (Springer, New York, 1999).

[9] P. F. Smith, 'Modules with many homomorphisms', J. Pure Appl. Algebra 197 (2005), 305-321.

[10] B. Stenstrom, Rings of Quotients (Springer, New York, 1975).

[11] K. Varadarajan, 'Dual Goldie dimension', Comm. Algebra 7 (1979), 565-610.

[12] R. Wisbauer, Foundations of Module and Ring Theory (Gordon and Breach, Philadelphia, PA, 1991).

[13] J. M. Zelmanowitz, 'Correspondences of closed submodules', Proc. Amer. Math. Soc. 124 (1996), 2955-2960.

[14] Z. P. Zhou, 'A lattice isomorphism theorem for nonsingular retractable modules', Canad. Math. Bull. 37 (1994), 140-144.

\section{B. AMINI, Department of Mathematics, College of Sciences, Shiraz University,}

Shiraz 71454, Iran

e-mail: bamini@shirazu.ac.ir

M. ERSHAD, Department of Mathematics, College of Sciences, Shiraz University, Shiraz 71454, Iran

e-mail: ershad@shirazu.ac.ir

H. SHARIF, Department of Mathematics, College of Sciences, Shiraz University, Shiraz 71454, Iran

e-mail: sharif@susc.ac.ir 